\title{
Numerical Studies of Astrophysical Objects at Fesenkov Astrophysical Institute (FAI)
}

\author{
Assylkhan Bibossinov ${ }^{1}$, Denis Yurin ${ }^{2}$, Chingis Omarov ${ }^{3}$
}

Address: Fesenkov Astrophysical Institute, Almaty, Kazakhstan

E-mail: ${ }^{1}$ bibossinov@live.com, ${ }^{2}$ d.jurin@gmail.com, ${ }^{3}$ chingis.omarov@gmail.com

\begin{abstract}
Numerical studies of astrophysical objects are a relatively new direction in Fesenkov Astrophysical Institute (FAl) and is mainly represented by the Laboratory of Cosmology, Stellar Dynamics and Computational Astrophysics. The lab seeks to understand the evolution of gravitating systems at various scales - from star clusters to galaxies to large-scale structure of the universe as a whole, and tackles these problems both through analytical methods and through numerical simulations. The particular focus is on numerical simulations of star clusters, especially those found in active galactic nuclei - this is a topic of oldestablished collaboration with colleagues from Astronomisches Rechen-Institut (Heidelberg) and National Astronomical Observatories of China (Beijing). The prominent example is STARDISK project dedicated to the numerical research of active galactic nuclei as multicomponent systems composed of compact stellar cluster, gaseous accretion disk and a supermassive black hole. It is demonstrated that an accretion disk can noticeably decelerate stars and thus enhance the accretion rate onto the black hole. In 2013 FAl hosted the MODEST-13 International Workshop dedicated to modeling of star clusters. Recently a new project has been approved aimed at construction of triaxial equilibrium $\mathrm{N}$-body systems that can be of great help in various numerical experiments with disk galaxies. There are also long standing plans to perform cosmological simulations of large scale structures to test a new approach to dark matter and energy actively developed at FAI. For numerical calculations, FAl has a small, but growing computer cluster consisting of several high-performance computing servers equipped with computational GPU cards.
\end{abstract}

Keywords: Numerical simulations, star clusters, galaxies, dark matter, neutrino

\section{Introduction}

Unlike most other disciplines in natural science, astronomy is not a 'laboratory science', because nobody can directly experiment with objects in the sky, no one can rearrange them. However, now that we have entered the era of advanced computer simulations, one can create a synthetic universe in a computer and "move" stars and galaxies in it. 
Numerical experiments allow us to decompose complex physical processes into their components, and in this way to derive their properties and their roles in the system as a whole. Moreover, the results of computer simulations can be observed in great detail over the entire phase space at any angle and as close as we like. This offers an unprecedented opportunity to see, for example, how exactly the large-scale structure of the Universe emerged from the primordial noise, how razor thin galaxies are able to survive despite the hazardous merging, or how processes like the radial migration of stars in disk galaxies affect the properties of individual galaxies. Moreover, the numerical modeling started to demonstrate that it may have also predictive power, it is in principle possible to discover in simulated sets a physical phenomenon that have not been detected yet in observational data.

Nowadays, a computational astrophysicist is as likely to be found puzzling over the engineering of a complex computer code and struggling to make it run on a computer cluster than to be found working with mathematical equations on paper (or chalkboard).Publications analysis shows that the number of astrophysical research works employing computer simulations and automated processing of large data sets continues to grow (Heng 2014),making it of prime importance for a modern astrophysical institute to enter the field of numerical studies and to have a computational facility for it.

To this end, FAl has dedicated research group (the Laboratory of Cosmology, Stellar Dynamics and Computational Astrophysics) that primarily works in the field of computation astrophysics. The group has carried and carrying out several prominent projects. Among them are the STARDISK project dedicated to the numerical research of active galactic nuclei, the GALIC 3D project aimed at construction of triaxial equilibrium $\mathrm{N}$-body models in collision less equilibrium, and the project devoted to investigation of the galaxies evolution within some non-traditional cosmological models.

For numerical calculations, the FAI has a small computer cluster consisting of several high-performance computing servers equipped with computational $\mathrm{PCl}$ cards.

\section{Laboratory of Cosmology, Stellar Dynamics and Computational Astrophysics}

As the lab name implies, the group seeks to understand the evolution of gravitating systems at various scales - from star clusters to galaxies to large-scale structure of the universe as a whole, and tackle these problems both through analytical methods and through numerical simulations with the help of computational technologies. 
At smaller scales, the group's particular focus is on numerical simulations of star clusters, especially those found in active galactic nuclei - the topic of our oldestablished collaboration with colleagues from Astronomisches Rechen-Institut (Heidelberg) and National Astronomical Observatories of China (Beijing). In 2013 the group had hosted the MODEST-13 International Workshop dedicated to modeling star clusters.

At larger scales, the dominant factors in the evolution of gravitating systems are dark matter and dark energy, and at this scale the group are interested in the origin and nature of this invisible stuff that makes up $95 \%$ of the universe. As well known, despite its great success in matching many astronomical observations, the standard cosmological \CDM-model still lacks firm experimental evidence for the existence of its underlying particles such as WIMPs. Therefore, alongside with the research within the $\Lambda C D M$ cosmology, the group also try to develop alternative models employing only experimentally known particles and fields.

The group is the main user of the computer cluster at FAI.

\section{STARDISK Project}

STARDISK is the joint project between FAI (Almaty) and Astronomisches RechenInstitut (Heidelberg). The project is sponsored by the Volkswagen Foundation. It aims at the numerical researches of active galactic nuclei as multicomponent system composed of compact stellar cluster, gaseous accretion disk and the black hole. Supermassive black holes are located in most if not all centers of galaxies, including our own. Their growth due to interaction with and accretion of surrounding gas and stars is one of the most interesting and important fields in astrophysics nowadays. New space and ground based telescopes of present (Hubble Space Telescope, VLT, Chandra, XMM Newton) and future generations (Next Generation Space Telescope) provide a large amount of new high-resolution data on morphology and kinematics of stellar and gaseous systems near black holes. Since the which the evolution of stars (gravitating mass points) with a central potential (black hole) and a gaseous disk is followed by direct modelling of stellar orbits. The project examines a special topic in this area, namely the interplay between stellar dynamical two-body relaxation and star-gas interactions due to a central accretion disk.

To facilitate direct $\mathrm{N}$-body simulations this project relies on special purpose hardware equipment like GRAPE and more recently Nvidia Tesla cards which, together with standard computers, provides an extremely competitive computing systems for direct $\mathrm{N}$-body simulations, with very good price-performance ratio. Both 
GRAPE and GPU cards are also used to equip computer clusters so that aparallelization of the task further speeds up the calculations. To employ massively parallel computing on special hardware for direct integration of dense stellar systems the two N-body codes have been used NBODY6++ (based on the family of codes developed by S.Aarseth) and phiGRAPE/GPU.

The project resulted in the creation of a small computer cluster at FAl, and also led to internships of young researchers at AstronomischesRechen-Institut (Heidelberg) where they got experience with high performance computing and with $\mathrm{N}$-body codes.

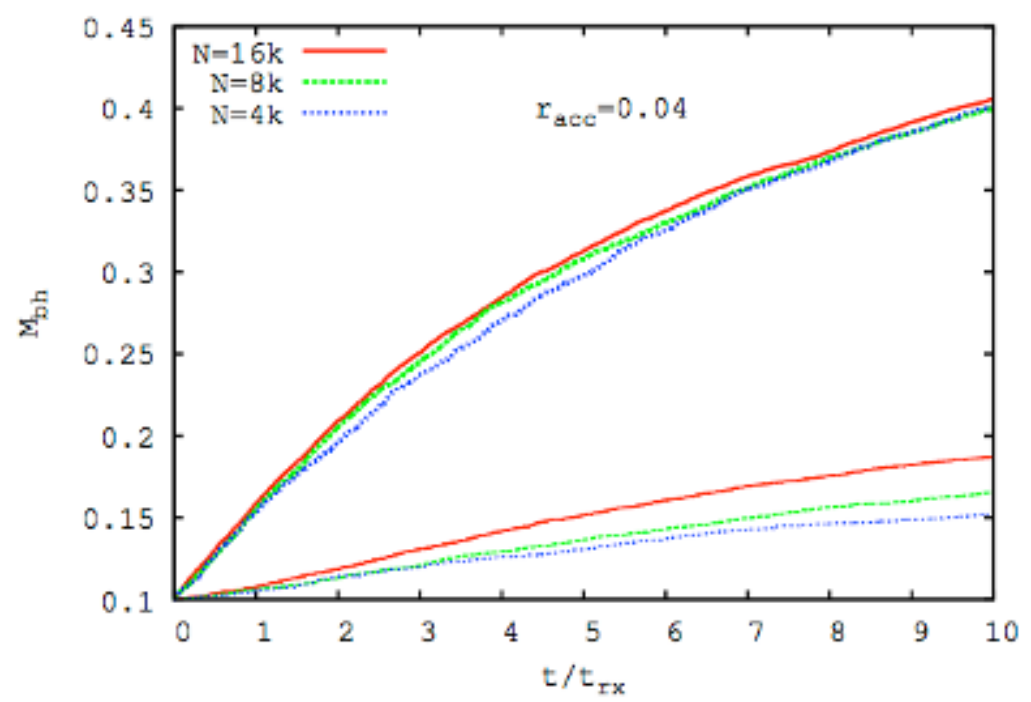

Figure 1. Supermassive black hole growth with (upper thick lines) and without (lower thin lines) dissipative force for specific accretion radius racc $=0.04$

The main finding of the project is that an accretion disk can indeed decelerate stars and thus enhance the accurate rate onto the black hole. This and some other results have been published in peer reviewed journals (Just et al. 2012).

\section{GALIC 3D Project}

The principal difficulty in performing numerical experiments with galaxies is related to generating model galaxies in unperturbed equilibrium state with given properties in the form of initial conditions to be used in simulation codes. The point is that real galaxies, are typically found in high-equilibrium state and represent actually near perfect collision less systems. Therefore, their dynamics is described by stationary solution of collision less Boltzmann equation (also known as Vlasov equation) coupled with Poisson equation. This solution yields the so called distribution function (DF) that accurately describes the distribution of mass and velocities at each point of the system. Finding DF with prescribed properties represents a mathematical 
problem of enormous complexity which is currently solved only for few simple and mainly spherically symmetric density distributions. In the absence of exact solutions, initial conditions for galaxies in numerical simulations are generated with models based on approximate solutions to Vlasov-Poisson equation. Such galaxies are subject to intrinsic instabilities which might interfere with the results of control experiments. Hence, it might be difficult to decide if some changes observed in a simulated galaxy are due to external effects or due to internal instabilities.

Recently, a significant progress has been made in solving this issue. In 2014, Denis Yurin and Volker Springel had created a principally new numerical method (Yurin \& Springel 2014) that allowed to generate model galaxies in essentially perfect equilibrium with prescribed density distribution and velocity structure. The method is based on the idea of iterative modification of initial particle velocities in order to superimpose their orbits so that they produce given density distribution and velocity anisotropy. With that, initial particle positions remain unaltered and fixed according to the target density distribution. Direction of velocity shift is defined by merit functions that measure the difference between the current and target density distributions, as well as between current and target velocity structures, with subsequent variation of velocities that minimize these merit functions. On basis of this method, authors created a computer code, dubbed as GALIC, to generate initial conditions for GADGET-2 code. The GALIC code was made publically available and could be freely used in scientific research by anyone. Up to date, it has been used in more than a dozen of independent research works, see, e.g., (Aumer \& Schoenrich 2015, Moetazedian \& Just 2016, Pardy et al. 2016, Laporte et al. 2018, Wu et al. 2016).

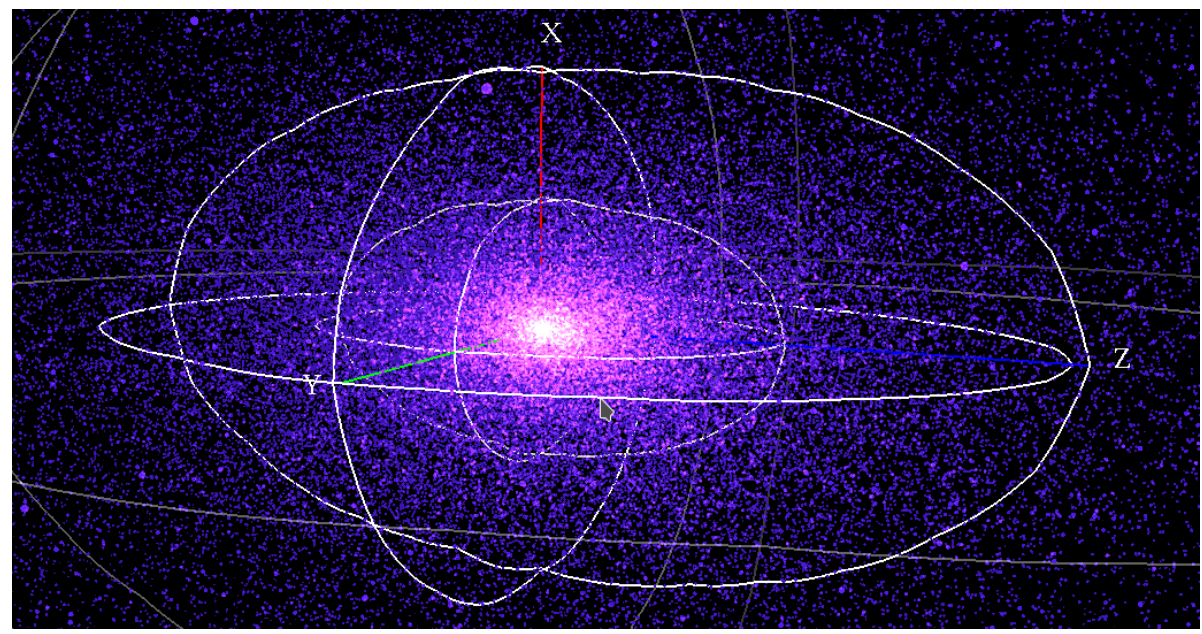

Figure 2.Open $G L$ visualization showing in $3 D$ the particle distribution of triaxal $n$-body system consisting of $128 \mathrm{k}$ particles with $\mathrm{X}: \mathrm{Y}: Z$ axis ratio $0.5: 1: 2$. White lines represent sis density contours. 

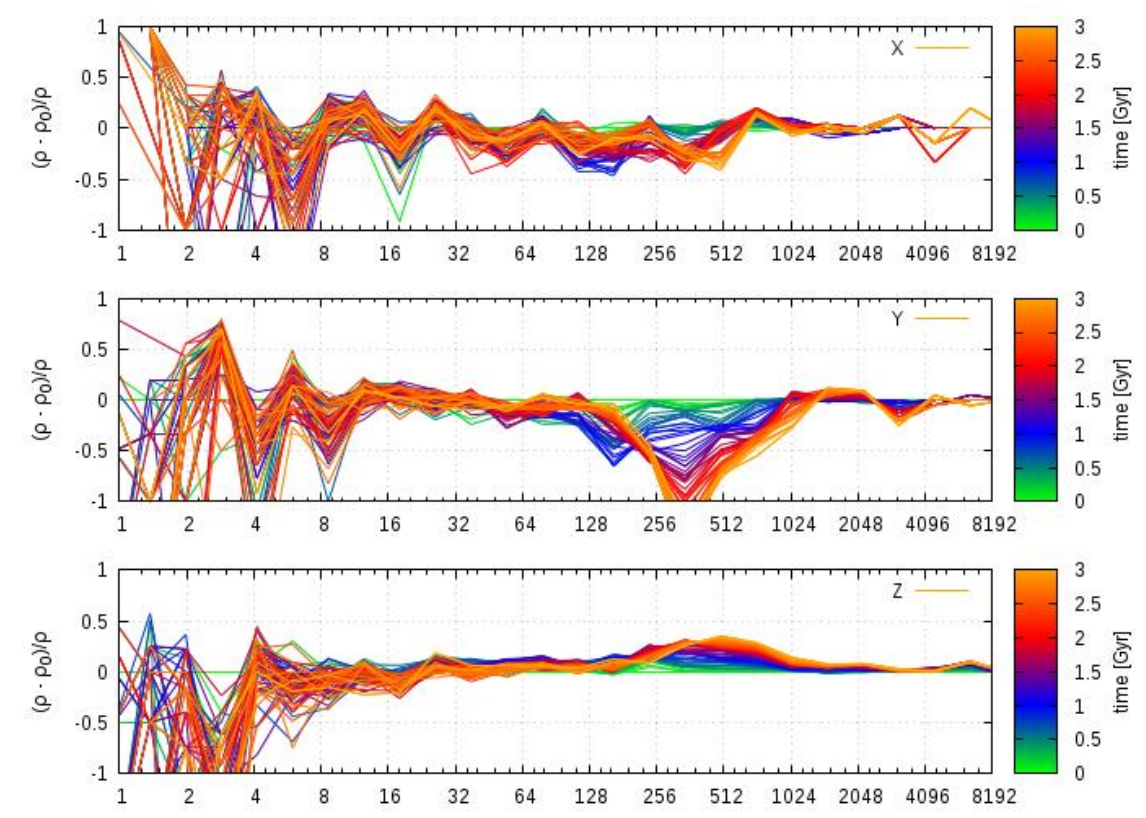

Figure 3. Density profile changes of the triaxial ellipsoid showed at Figure 2 along its major axes after its particle velocities were initialized with GALIC 3D and then evolved over time with GADGET code (preliminary results). While density distribution along minor $(X)$ and major $(Z)$ axes worked out to be relatively stable, over middle axis $(\mathrm{Y})$ there is significant density deviation from original profile at 128 $1024 \mathrm{kpc}$.

While the GALIC code has been in use, a number of proposal for its further improvement have been made. The most in-demand request is concerned with the development of a fully triaxial version of the code. Currently, GALIC might generate only axisymmetric systems, which limits its usage in problems involving triaxial systems such as dark matter halos, bars, spiral arms and others. This limitation is not fundamental but is conditioned by certain technical difficulties of adaptive discretization of three-dimensional space and necessity to close Jeans equations for triaxial systems. Apart from that, the code was parallelized only with Message Passing Interface (MPI), without using vector instructions SSE/AVX, and without CUDAparallelization for GPU-cards, making the code less effective in employing computational resources. Also, during code exploitation it was found that velocity anisotropy in GALIC is measured at initial moment without averaging over time as done for density distribution, which might lead in certain cases to generating initial conditions somewhat different from those expected. Thus, there is a significant necessity in updating and further development of the GALIC code. In 2018 the corresponding proposal to extend GALIC code to triaxial systems, solve discovered problems and parallelize it for GPU got funding from Aerospace Committee of the Ministry of Defense and Aerospace Industry of Kazakhstan in form of GALIC 3D project on 2018-2020.Already at the end of the first year, the project succeeded to 
create relatively stable triaxial $n$-body system with prescribed density distribution (see Figure 3).

\section{Cosmological simulation within alternative dark matter models}

During 2012-2014 FAPHI held a grant project funded by the Ministry of Education and Science of Kazakhstan, and devoted to the investigation of evolution of galaxies within some non-traditional cosmological models. In particular, the cosmological group in FAPHI strives to revive the (historically) first dark matter model - the neutrino dark matter - but with an additional assumption that neutrinos might exist in special tachyonic states (Makukov et al. 2015, Mychelkin \& Makukov 2015). This assumption does not contradict experimental data, since the velocity excess over the speed of light is so tiny that no currently existing experiments can probe the difference. At the same time, this extra assumption saves the neutrino dark matter model from many defects of the traditional neutrino dark matter model, primarily because in this case there is no constraint on neutrino number density and so it might be chosen to fit observations.

Within the framework of this alternative model, we had performed computer simulations of evolution of galaxies. The tachyonic neutrino model suggests that dark matter haloes have isothermal density profiles with the central singularity smoothed-out (Makukov et al. 2015). We used the GADGET2 code to simulate a twocomponent galaxy (having disk and bulge) embedded into an isolated dark matter halo characterized by the isothermal profile, during 1 Gyr. Figure 4 shows the visual representation of the evolving galaxy. The main finding is that the resulting rotation curves tend to be saturated far-out and fit the observational data well (see the grey curve in Figure 5).
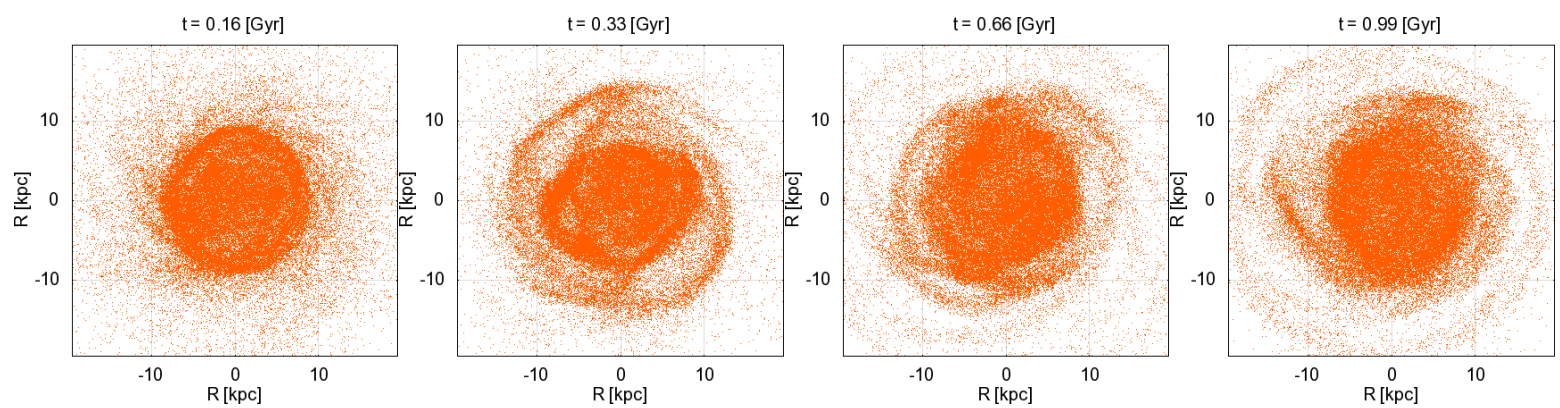

Figure 4. Face-on view of the simulated galaxy at various moments during evolution. 
In future, we are planning to perform large-scale cosmological simulations to test how the proposed alternative dark matter model fits with observational data on matter clumping.

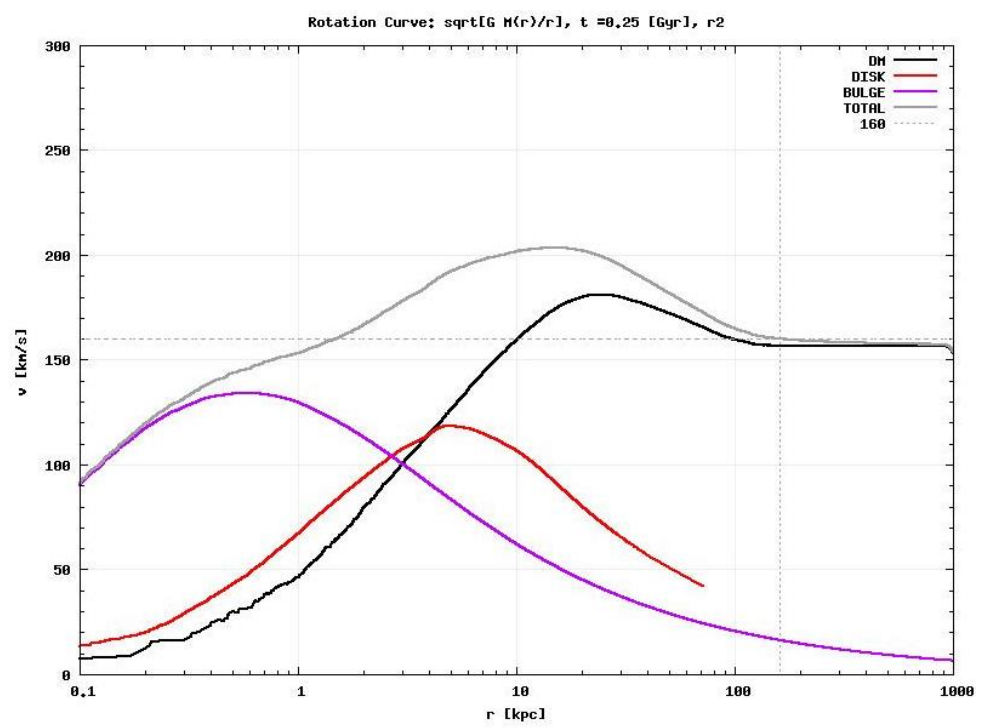

Figure 5. Rotation curves for a simulated galaxy. Black: only dark matter, red: only disk, magenta: only bulge, grey: total.

\section{Computer Cluster at FAI}

Development of a computational codes, debugging and testing them as well as carrying out production runs of actual simulations imply massive computations which are impossible without related high-performance computing infrastructure. In 2008 a mini cluster had been assembled in FAI which was then successfully used in several scientific projects, including the STARDISC project, World Space Observatory Ultraviolet and a number of others. Since then the cluster growth significantly supplied by different projects and sources including Maecenas's support. Currently the computer cluster at FAI consists of several high-performance computing servers equipped with computational $\mathrm{PCl}$ cards allowing to perform various parallelized jobs on multiple CPUs and GPUs. The theoretical single precession performance of the cluster now peaks at 5 TFLOPS for CPU operations (68 cores / 120 threads) and 58 TFLOPS for GPU (17536 CUDA cores).

We choose to develop our own computing infrastructure, instead of using remote date centers of collective use because of two main reasons. First, this is way more cost-efficient, and, second, it is much more reliable. We can build exactly what we need and use it when we need it. 
At the moment, the cluster runs at full load on $24 / 7$ basis and FAI is actively seeking for new opportunities to extend it further to even larger computational system.

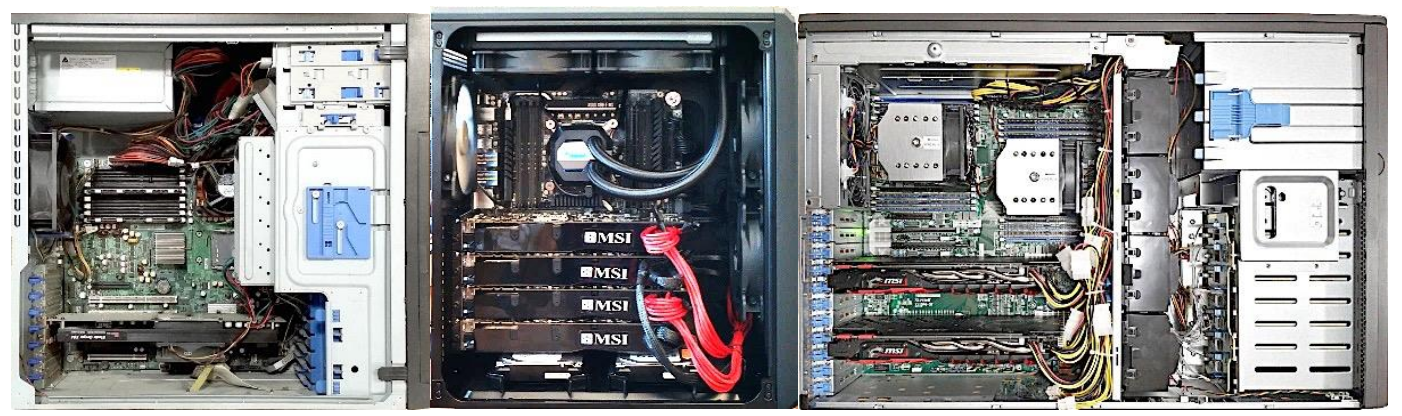

Figure 6.Examples of computational servers used in computer cluster at FAI. Left image: 8-core GRAPE-card server used in STARDISK project (2009); middle image: 16-core desktop-based server for

CPU/GPU computing (2015) and right image: 48-core CPU/GPU server bought just recently in framework of GALIC 3D project (2018).

\section{Summary}

The exponential growth of computer performance in the recent past has dramatically increased the capabilities of numerical methods. Now, when computer simulations can mimic reality in an increasingly realistic fashion, we can carry out controlled experiments of synthetic astrophysical or other processes, and take their results as an approximate reflection of real experiments. This is particularly fascinating in astronomy, were the corresponding real experiments can actually not be carried out.

It is also indicative, that in common terminology in the field of computational astrophysics, simulated results now are often described as being "empirical" data, a term usually reserved for natural phenomena rather than for numerical models of nature. It is not rare to hear that simulated data are referred to as "data sets", seemingly placing them on an equal footing with observed natural phenomena.

There is no doubt however that when interpreting numerical experiments, we have to be careful. To use a simulation as a laboratory, one has to understand its limitations - otherwise, one may wrongly identify an artifact as a result. For example, numerical oscillations or enhanced viscosity that arise from errors in numerical schemes could be easily be mistaken as physical effects.

The danger of blurring this distinction is probably small in reality, even though virtual laboratories offer opportunities for experimentation and observation that in some sense even go beyond what is possible in any real physical laboratory. In a virtual laboratory on the computer, we can turnoff or turn on any processes we want, analyzing how they affect each other. Or we can change their properties (even into 
unphysical regimes) in order to understand what role they play in detail. We can trace back in time the evolution of any object to see how its properties emerged. We can observe a process under any spatial and time angle, unrestricted by sensitive limitations of instruments.
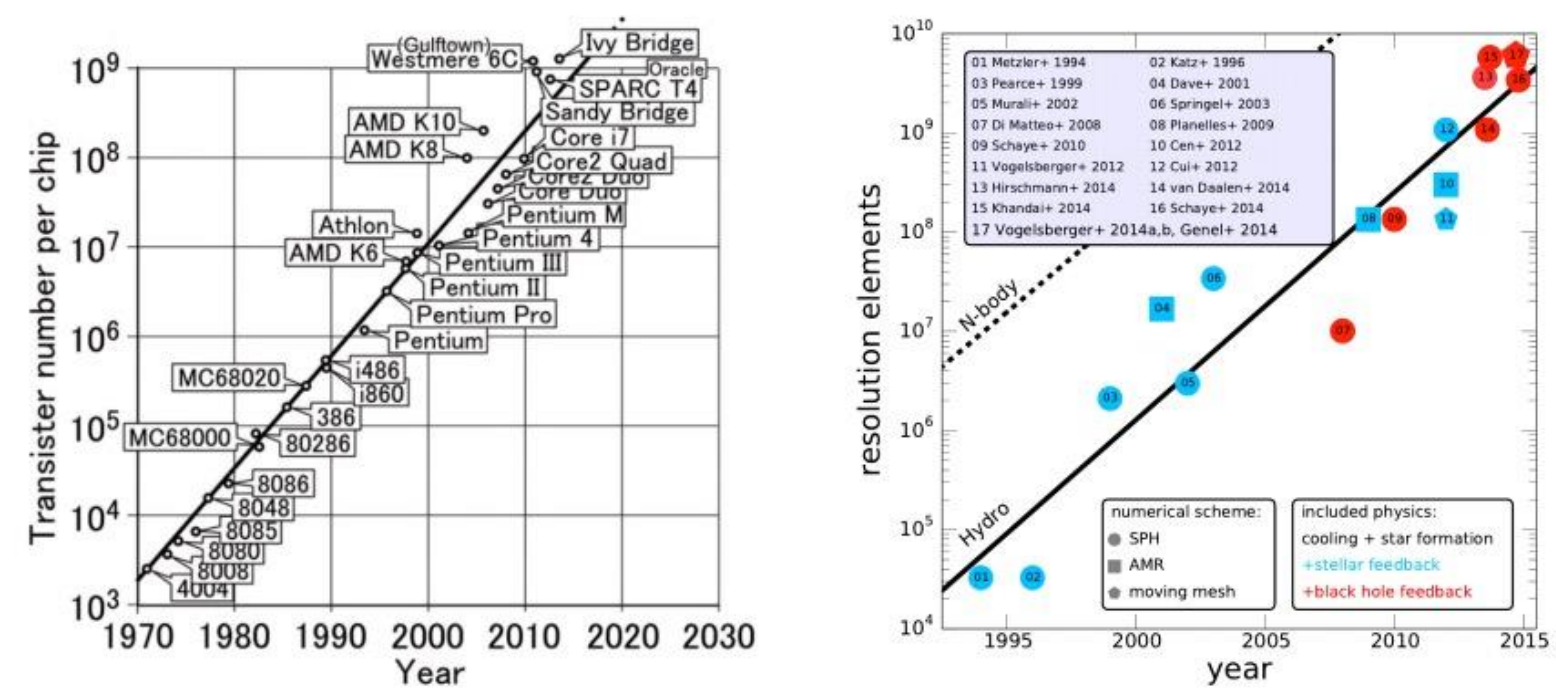

Figure 7. Left image: Moore's Law for microprocessors, showing that the number of transistors in microchips doubles approximately every two years. Right image: Growth of resolution elements in cosmological simulations as a function of publication year (adopted from (Genel et al. 2014)). This shows the same tendency to double roughly every two years.

Since Moore's law (Moore 1965) still holds true (see Figure 7), continuing the trend over the last 40 years, we can hope that the accuracy and level of detail reached in numerical simulations will still continue to grow (Genel et al. 2014). And consequently, the reliability of virtual experimentation should continue to grow as well.

To bring a numerical model to a life and use it scientifically, one needs to write computer code that realizes all operations prescribed by the numerical model. Making good use of the rapidly growing power of modern computer hardware is a great challenge for astrophysics, requiring also good knowledge in computer science.

All these together make us to believe that numerical studies of astrophysical objects and related technologies and knowledge become an absolute need for the nowadays success at international scientific level. For us, it gets clear that developing computational facilities and conducting computational studies becomes of the same importance as development of observational instruments and conducting observations. That is why, the FAl is trying to keep up with this tendency by running the computational astrophysics group and building up own high-performance 
computing infrastructure and corresponding competences. Success and international recognition of a number of projects carried out in the field of computational astrophysics ensured us that we stepped the right way to go.

We glad to see that Kazakhstan National Science Report of 2015 recognizes the necessity to create a supercomputer network and provide access to it to scientific organizations and researchers as one of the major directions in the development of the national science.

\section{References}

Aumer M., Schoenrich R., 2015, MNRAS, 454, 3166

Genel S., et al., 2014, MNRAS, 445, 175

Heng K., 2014, American Scientist, 102, 174

Just A., Yu. D., Makukov M., Berczik P., Omarov C., Spurzem R., Vilkoviskij E.Y., 2012, ApJ, 758, 51

Laporte C.F.P., Gomez F.A., Besla G., Johnston K.V., Garavito-Camargo N., 2018, MNRAS., 473, 1218

Makukov M.A., Mychelkin E.G., Saveliev V.L., 2015, 9th Alexander Friedmann International Seminar on Gravitation and Cosmology / 3rd Satellite Symposium on the Casimir Effect. St Petersburg, RUSSIA,

Moetazedian R., Just A., 2016, MNRAS, 459, 2905

Moore G., 1965, Electronics Magazine, 38

Mychelkin E.G., Makukov M.A., 2015, International Journal of Modern Physics D, 24, id. 1544025

Pardy S.A., D'Onghia E., Athanassoula E., Wilcots E.M., Sheth K., 2016, ApJ, 827, 149

Wu Y.T., Pfenniger D., Taam R.E., 2016, ApJ, 830, 111

Yurin D., Springel V., 2014, MNRAS, 444, 62 